\title{
Imaginarios en torno al curso de Teoría Contable: el caso del programa de Contaduría Pública de la Universidad de la Amazonia*
}

\author{
Carlos Alberto Gómez-Cano ${ }^{\star *}$, Verenice Sánchez-Castillo ${ }^{\star * *}$, Diana Ali García-Capdevilla ${ }^{\star * *}$, \\ Lorena García-Andrade ${ }^{\star * * * *}$, Yuri Alejandra Barrera-Rojas $s^{\star * * * *}$
}

* Artículo de investigación

** Contador Público, Administrador Público, Especialista en Pedagogía y en Gestión Pública, candidato a Magíster en Ciencias de la Educación. Docente del Programa de Contaduría Pública de la Universidad de la Amazonia. Grupo de Estudio de Futuro en el Mundo Amazónico (GEMA).

Correo electrónico: carlosgomez325@gmail.com

*** Docente-investigadora de la Universidad de la Amazonia.

Correo electrónico: ve.sanchez@udla.edu.co

**** Contador Público, Especialista en Gerencia Tributaria y en Gestión Pública, Magíster en Mercadeo. Docente del Programa de Tecnología en Gestión de Mercados, Universidad de la Amazonia.

Correo electrónico:

dianaaligarciacapdevilla@gmail.com

$* * * * *$ Estudiante del Programa de Contaduría Pública de la Universidad de la Amazonia, integrante del semillero de investigación Faccea. Correo electrónico:

semillerofaccea@gmail.com

$* * * * *$ Estudiante del Programa de

Contaduría Pública de la Universidad

de la Amazonia, integrante del

semillero de investigación Faccea.

Correo electrónico:

semillerofaccea@gmail.com

Recibido: 19 de julio del 2016

Aprobado: 22 de enero del 2017

Cómo citar este artículo: Gómez-Cano, C. A., Sánchez-Castillo, V., García-Capdevilla, D. A, García-Andrade, L. y Barrera-Rojas, Y. A. (2017). Imaginarios en torno al curso de Teoría Contable: El caso del programa de Contaduría Pública de la Universidad de la Amazonia. Cooperativismo \& Desarrollo, 111(25), 81-89. doi: https://doi. org/10.16925/co.v25i111.1873

\section{Resumen}

Introducción: se presenta una revisión crítica en torno a la educación contable en Colombia, y de la importancia de la teoría contable en la formación de los contadores públicos. Se evidencia con esto la necesidad de articular a los integrantes de las comunidades académicas, a fin de fomentar un desarrollo disciplinar. Metodología: el paradigma orientador fue el empírico analítico, y el tipo de investigación exploratorio descriptivo, con la aplicación de encuestas. Discusión y resultados: fruto de los resultados de la investigación quedó al descubierto la errada interpretación que se tiene sobre teoría contable, las falencias del accionar docente y la apatía de los futuros contadores públicos sobre este importante tema en la construcción de una disciplina contable sólida. Además, se hace hincapié en la necesidad de intervención por parte del programa, a fin de generar procesos de trasformación académica. Conclusiones: se deben hacer exhaustivas revisiones sobre el curso de teoría contable, y la forma como se articula con otros espacios académicos, situación que demanda la intervención de todos los integrantes de la comunidad universitaria.

Palabras clave: comunidad universitaria, educación, imaginarios, teoría contable. 


\title{
Imaginaries revolving around the Accounting Theory course: The case of the Public Accounting program of the Universidad de la Amazonía
}

\begin{abstract}
Introduction: A critical review about accounting education in Colombia, and the importance of accounting theory in the formation of public accountants is presented. This demonstrates the need to articulate $i$ members of the academic communities in order to foster disciplinary development. Methodology: The guiding paradigm was analytical empirical, and the research type was exploratory and descriptive, with survey application. Discussion and results: As a result, the research revealed the wrong interpretation of accounting theory, the shortcomings of teacher action and the apathy of future public accountants on this important issue for the construction of a solid accounting discipline. In addition, emphasis is made on the need for the program's intervention in order to generate processes of academic transformation. Conclusions: Exhaustive reviews on the course of Accounting Theory, and how it articulates with other academic spaces must be done. This demands the intervention of all members of the university community.
\end{abstract}

Keywords: university community, education, imaginaries, accounting theory

\section{Imaginários sobre o curso de teoria contábil: o caso do programa de contabilidade pública da Universidad de la Amazonia}

\section{Resumo}

Introdução: apresenta-se uma revisão crítica sobre o ensino de contabilidade na Colômbia e a importância da teoria contábil na formação dos contadores públicos. Evidencia-se, com isso, a necessidade de articular os integrantes das comunidades acadêmicas a fim de fomentar um desenvolvimento disciplinar. Metodologia: o paradigma orientador foi empírico-analítico, e o tipo de pesquisa, exploratório-descritivo, com a aplicação de questionários. Discussão e resultados: fruto dos resultados da pesquisa, evidenciou-se a errada interpretação que se tem sobre a teoria contábil, as falências do fazer docente e a apatia dos futuros contadores públicos sobre esse importante tema na construção de uma disciplina contábil sólida. Além disso, enfatiza-se a necessidade de intervenção por parte do programa para gerar processos de transformação acadêmica. Conclusões: deve-se fazer exaustivas revisões sobre o curso de teoria contábil e a forma como se articula com outros espaços acadêmicos, situação que demanda a intervenção de todos os integrantes da comunidade universitária.

Palavras-chave: comunidade universitária, educação, imaginários, teoria contábil. 


\section{Introducción}

\section{La educación contable en Colombia}

La formación de los contadores públicos profesionales en Colombia, a juicio de algunos expertos en el tema, atraviesa una profunda crisis. Al punto, por ejemplo, según Ospina (1994), de que los esfuerzos están dados en la obtención del título profesional, de modo que se rezagan el aprendizaje y la formación de competencias como objetivos básicos del proceso educativo. En esta línea, Franco et al. (2002) consideran que la ausencia de reflexión y cuestionamientos en los programas académicos ha generado paquidermia en los profesionales ante situaciones dinámicas y contradictorias de nuestro actual contexto.

En la misma línea, González (2008), y Ordóñez (s. f.), consideran que las deficiencias existentes en la educación contable de Colombia obedecen a la errada visión de algunos docentes y estudiantes sobre la contabilidad, al considerarla como una técnica eminentemente práctica, de manera que reducen sus discusiones académicas a un subsistema, bajo métodos rígidos y determinados por la repetición y la reproducción sin ningún tipo de análisis. Para Martínez (2008), esta situación es el resultado de los modelos pedagógicos que privilegian los modelos tradicionales y técnicos de la contabilidad, y logran así tan solo el desarrollo de competencias simples.

Aunado a lo anterior, Avellaneda y Avellaneda (s.f.) sostienen que, sin desconocer algunos esfuerzos de comunidades y grupos académicos que luchan por mejorar las condiciones de la disciplina contable, el desarrollo de la educación contable no ha superado crisis ontológicas y epistemológicas. De igual forma, se adolece de actualización de los métodos de enseñanza, pues los métodos tradicionales en una sociedad moderna no encajan, ni son funcionales para la generación en formación, ya que premian la instrumentalización y la mercantilización por encima de la construcción del conocimiento (Barone y Mella, 2003; Quirós, 2006).

En tal sentido, siguiendo a Ospina (2009), la educación contable debe asumir varios procesos de transformación, los cuales deberán atender contextos específicos y particulares, además de inyectar nuevas dinámicas en los actores del proceso educativo para que se empoderen de su rol y generen nuevos escenarios y dinámicas. Acorde con esta posición,
López (2012) propone que además del incentivo por las competencias en la educación contable, se debe privilegiar el componente ético como escenario estratégico en el repensar de la profesión.

\section{Importancia de la formación en teoría contable}

Tal y como se abordó en el acápite anterior, para el desarrollo científico de la contabilidad, es indispensable la transformación de las prácticas en la educación contable, por lo cual se deberán privilegiar espacios académicos que fomenten la disertación, el cuestionamiento, la investigación y el desarrollo científico. Sobre el particular, Ortiz (2010) expone que es indispensable para los profesionales de la contabilidad el desarrollo de habilidades y competencias que les permitan interpretar los fenómenos sociales que germinen a través del tiempo.

De ahí que, siguiendo a Franco (1997), se deben privilegiar espacios de formación en torno a la teoría contable, pues desde esos escenarios la práctica contable se puede evaluar y dirigir al desarrollo de nuevos procesos. En concordancia, Grajales y Cuevas (2010) exponen que la teoría contable juega un papel protagónico en la formación de los contadores públicos, pues fomenta la discusión científica de la disciplina, sin embargo, plantean la necesidad de repensar los cursos y su dinámica académica al interior de las universidades.

Con relación a lo anterior, Gómez (2006) asiente la necesidad de provocar y repensar los espacios de formación en teoría contable, a fin de, según Dextre (2011), fomentar cultura investigativa, desarrollo humano y consolidación científica de la contabilidad. En palabras de Pinto (2010, p. 2): "requerimos evaluar, revisar, y ajustar nuestras concepciones de la contabilidad y de la Teoría Contable, así como las metodologías y enfoques con que las enseñamos o abordamos en el aula".

En tal sentido, el presente manuscrito presenta los resultados de un proceso de investigación realizado en la Universidad de la Amazonia, sobre las realidades, concepciones e imaginarios de los estudiantes del programa de Contaduría Pública con respecto al curso Teoría Contable. Bajo este entendido, se presentan dos grandes resultados: la percepción de los estudiantes, y los aspectos a mejorar, a fin de que el mencionado espacio académico cumpla con su rol protagónico como gestor de escenarios de reflexión y desarrollo disciplinar. 


\section{Metodología}

\section{Localización}

El presente estudio se realizó en la Universidad de la Amazonía, localizada en el municipio de Florencia, departamento del Caquetá, al suroccidente de Colombia.

\section{Población y muestra}

La población objeto del estudio fueron los estudiantes del programa de Contaduría Pública de la Universidad de la Amazonia que se encontraban cursando entre el vi y el x semestre, y aquellos que se encontraban desarrollando su opción de grado, a corte del iI semestre académico del 2015, pues son ellos quienes reciben o recibieron el curso denominado Teoría Contable, estipulado dentro del vi semestre del mencionado espacio académico. Así las cosas, el presente trabajo fue de corte longitudinal y centró su atención durante el periodo comprendido entre el 2013 y el 2015.

\section{El enfoque metodológico}

El enfoque de la presente investigación es empírico analítico, en razón a que la realidad se estudia a través de variables cuantificables que buscan producir regularidades que permitan proposiciones (Gutiérrez, 2014). Es de corte exploratorio y descriptivo, porque aborda situaciones que ocurren en condiciones naturales, más que aquellas que se basan en asuntos experimentales (Beltrán, Gómez y Figueroa, 2013), las cuales permiten indagar acerca de la realidad del estudiante de Contaduría Pública de la Universidad de la Amazonia.

\section{El método}

Si se tiene en cuenta cómo, más que el levantamiento de los datos, al identificar imaginarios se hace necesario hablar de la producción de estos en un diálogo con los diferentes actores involucrados (Sánchez et al., 2016), se realizó el método de trabajo que se describe a continuación.

\section{Caracterización de la población objeto del estudio y el contexto de estudio.}

En esta parte se empleó la técnica arqueo de archivo, y se consultaron bases de datos de los estudiantes que habían visto el curso de Teoría Contable durante el periodo de agosto a diciembre del 2015. De igual forma, se consultaron los documentos internos, las guías de trabajo del curso de formación, las formas de evaluación y la bibliografía empleada para la orientación del curso, en un periodo retrospectivo de dos años en el que los estudiantes objeto del estudio recibieron este curso de formación. Esta información se colocó en plantillas de doble entrada y se resaltó el nombre del documento, la localización y el hallazgo.

\section{Identificación de la población y la muestra.}

Los estudiantes que a la fecha de la investigación estaban cursando la asignatura de Teoría Contable, así como los estudiantes que ya la habían cursado -es decir, que cumplían los requisitos para informantes clave-, sumaron un total de 610 individuos, de los cuales fue posible encuestar al 82,13\%; esto significa que se contó con un nivel de confianza del $97 \%$, y un error estadístico de los datos solo del $2 \%$.

\section{Definición de las variables de análisis.}

Al tener en cuenta los resultados de la caracterización y los hallazgos en la revisión de las fuentes secundarias, en especial los documentos internos del programa del curso de Teoría Contable, se definieron como categorías para la pesquisa: las razones de la importancia del curso, el imaginario alrededor de sus objetivos, los aspectos curriculares, los factores a mejorar, el posible interés en realizar un posgrado en el área, y su desempeño profesional.

\section{Construcción y aplicación de los cuestionarios.}

De acuerdo con el conjunto de variables definidas, se elaboró un grupo de preguntas por cada uno de ellas, y se diseñó un cuestionario de 12 preguntas. Posteriormente, se consultaron los horarios de los respectivos estudiantes y se visitó cada aula de clase, en la que se aplicó, de manera personalizada, el cuestionario elaborado.

\section{Redacción de los hallazgos.}

La información elaborada se sistematizó en una base de datos, por medio de cuyas correlaciones se fue dando forma a la producción de los datos y la redacción de los hallazgos. 


\section{Resultados y discusión}

\section{Percepción en torno al curso Teoría Contable}

A manera de contexto, se tiene que el programa de Contaduría Pública, en la Universidad de la Amazonia, funciona gracias a la Resolución 8451 del 23 de julio de 2012, la cual fue expedida por el Ministerio de Educación Nacional, y por la cual se renovó el registro calificado del programa por siete años. Dentro del documento maestro, el plan de estudios establece 166 créditos académicos, distribuidos como se muestra en la tabla 1.

Tabla 1

Áreas del plan de estudios del programa de Contaduría Pública

\begin{tabular}{lll}
\hline \multicolumn{1}{c}{ Área } & \multicolumn{1}{c}{ Créditos } & $\%$ \\
\hline Contable y Financiera & 77 & 46 \\
\hline Cuantitativa & 15 & 9 \\
\hline Económico Administrativas & 37 & 22 \\
\hline Legal & 20 & 12 \\
\hline Humanística & 6 & 4 \\
\hline Electivas & 8 & 5 \\
\hline Investigativas & 3 & 2 \\
\hline TOTAL & 166 & 100 \\
\hline
\end{tabular}

Fuente: Tomado de http://www.udla.edu.co/v10/index.php/programas/profesionales/ contaduria-publica.html

Tal y como lo detalla la tabla 1, cerca de la mitad de los créditos académicos se concentran en el área Contable y Financiera, escenario donde de los 21 espacios académicos agrupados, tan solo uno, ubicado en el vi semestre del plan de estudios, obedece a la formación en teoría contable; los demás están relacionados con Contabilidad para el Sector Público y Privado, Costos, Finanzas, Proyectos, Auditoría, Presupuesto, y Revisoría Fiscal, entre otros.

Sobre el particular, Cortés (2006) expone que en la gran mayoría de los programas académicos de contaduría pública se le presta mayor atención al aspecto práctico e instrumental de la contabilidad, que al desarrollo del pensamiento crítico. Esta situación no es ajena al programa de Contaduría Pública de la Universidad de la Amazonia, pues de los 52 espacios académicos establecidos en su plan de estudios, tan solo uno está destinado a la formación en teoría contable, es decir, solo el $2 \%$ de los cursos exigidos para obtener su título profesional.

En tal sentido, con el propósito de develar las percepciones en torno al curso de teoría contable, se indagó sobre su importancia, y se encontró que el $94,8 \%$, lo que representa 475 estudiantes, considera que es un espacio académico importante; tan solo el 5,2\% considera que no es relevante para su formación profesional como contadores públicos. Sin embargo, al indagar sobre las razones que le daban importancia al curso, se encontró el panorama que se expone en la tabla 2 .

Tabla 2

Razones de importancia del curso Teoría Contable

\begin{tabular}{llc}
\hline \multicolumn{1}{c}{ Razón } & \multicolumn{1}{c}{ Cant. } & $\%$ \\
\hline Porque es prerrequisito de otros cursos. & 74 & 15,5 \\
\hline $\begin{array}{l}\text { Porque permite una nueva mirada a la } \\
\text { disciplina contable. }\end{array}$ & 370 & 77,4 \\
\hline $\begin{array}{l}\text { Es un nuevo estilo de clase y desarrollo de } \\
\text { temáticas. }\end{array}$ & 31 & 6,5 \\
\hline TOTAL & 475 & 100 \\
\hline
\end{tabular}

Fuente: elaboración propia con base en la encuesta y cálculos propios.

El anterior escenario deja en evidencia cómo, aunque la mayoría de la población considera que el curso es importante por su acción critica, aún existen quienes la conciben como una obligación académica más. Esto, según Marulanda, Piedrahitay Quintero (2011), obedece a un paradigma de ejecución sin sentido: hacer por hacer, entregar por entregar. Por otra parte, al cuestionar sobre el objetivo del curso, los estudiantes proporcionaron las respuestas que se presentan en la tabla 3.

Tabla 3

Objetivos del curso Teoría Contable

\begin{tabular}{lcc}
\hline \multicolumn{1}{c}{ Objetivo } & Cant. & $\%$ \\
\hline $\begin{array}{l}\text { Cambiar la percepción de lo instrumental, } \\
\text { hacia algo un poco más teórico. }\end{array}$ & 123 & 24,6 \\
\hline $\begin{array}{l}\text { Desarrollar en estudiante habilidades para } \\
\text { criticar, proponer y analizar situaciones de la } \\
\text { disciplina contable. }\end{array}$ & 332 & 66,3 \\
\hline $\begin{array}{l}\text { Consolidar lo instrumental de la disciplina } \\
\text { contable }\end{array}$ & 46 & 9,2 \\
\hline TOTAL & 501 & 100 \\
\hline
\end{tabular}

Fuente: elaboración propia con base en la encuesta y cálculos propios. 
La realidad encontrada deja en evidencia nuevamente un desconocimiento por parte de los estudiantes respecto a la importancia y el verdadero sentido del curso de Teoría Contable, pues aún persisten respuestas que giran en torno a lo instrumental de la contabilidad, situación que puede ser atribuida a que los estudiantes antes de tomar el curso han hecho un recorrido por cinco espacios académicos con fines exclusivamente técnicos. Bajo estas circunstancias, conviene retomar lo planteado por Giraldo (2010, p. 231), quien afirma:

Al parecer, el estudiante no ha entendido la función de la investigación y la epistemología en el avance cognoscitivo de la contabilidad, y mucho menos de la historia y la crítica; su impaciencia causa el efecto "anteojeras de caballo", lo cual impide tener miradas perpendiculares y circulares sobre las relaciones de la contabilidad y sus entornos.

Sin embargo, sería improcedente descargar toda la responsabilidad en los estudiantes, pues estos no son los únicos integrantes de la comunidad académica; también se debe cuestionar el actuar docente y las políticas institucionales. Sobre el particular, Ríos (1998) cuestiona el rol trasmisor del docente, lo que obliga al estudiante a convertirse en un receptor, situación que limita el avance académico y los procesos de enseñanza-aprendizaje. Bajo este entendido, conviene retomar lo planteado por González $(2008$, p. 1):

Si verdaderamente se quiere un avance de trascendencia en la educación contable, este debe fundamentarse desde el desarrollo del pensamiento crítico a nivel del aula, tanto en profesores como en estudiantes. La educación contable en Colombia está cambiando, y debe seguir en esta lucha, se hace corriente que en las aulas y en los pasillos de las universidades, profesores y estudiantes desaprueben los programas curriculares rígidos, los métodos de enseñanza autoritarios basados en la repetición, la reproducción del saber sin ningún tipo de análisis.

En línea con lo anterior, se indagó si al finalizar el curso de Teoría Contable habían generado una nueva percepción sobre la contabilidad, encontrándose que el 20,2\%, es decir, 101 estudiantes, respondieron de forma negativa; el restante $79,8 \%$ respondió de forma positiva. Este escenario, especialmente para quienes respondieron de forma negativa, según Rojas (1996), limita el papel protagónico de la teoría contable en la transformación de la contabilidad como disciplina.
Finalmente, la situación se hace más compleja al consultar a los estudiantes sobre el efecto del curso en su perfil profesional, pues de los 501 encuestados, el $27,5 \%$ consideró que no tuvo ningún efecto sobre su trasegar académico. En este contexto cobran validez los planteamientos de Rojas (2008, p. 266), quien afirma:

Muchos de quienes estudian y han estudiado contabilidad llegan a la universidad cargados de intereses meramente económicos por aprender a contabilizar y por participar en el mundo empresarial. Simplemente iniciaron sus estudios con el deseo de lograr una cualificación personal en su saber que en principio desconocen, pero del cual, suponen, permite en poco tiempo lograr una especialización que los inserta en el mercado laboral.

\section{Aspectos curriculares y de mejora para el curso Teoría Contable en el programa de Contaduría Pública de la Universidad de la Amazonia}

Ariza y Villasmil (2014) sustentan que la teoría contable se constituye en un medio para nutrir y promover el avance de la contabilidad como ciencia, razón que insta a las instituciones de educación superior a cualificar sus procesos formativos, promoviendo una visión crítica y reflexiva, a fin de atender positivamente las exigencias de la sociedad. Por su parte, Rueda, Pinzón y Patiño (2013) consideran que los programas de Contaduría Pública en Colombia deben realizar una revisión exhaustiva de sus currículos, amén de contar con programas que impacten los procesos sociales.

Dado lo anterior, se indagó en los estudiantes acerca de su percepción respecto a la ubicación del curso Teoría Contable dentro de la malla curricular y el espacio académico contemplado en el vi semestre. Se encontró que de los 501 encuestados, el 63,7 \% considera que debería ubicarse en los primeros semestres, a fin de generar las dos visiones de la disciplina de manera paralela —-técnica y científica-; el 28,1\% considera que la posición dentro del trasegar académico es adecuada; y, finalmente, el 8,2 \% considera que estaría mejor al final de la carrera, cuando se haya hecho un recorrido académico más riguroso.

Por otra parte, al indagar sobre los posibles aspectos de mejora del curso, el $22 \%$ de los encuestados respondió que, desde su juicio, no existen procesos para mejorar; empero, el restante $78 \%$ consideró que sí existen elementos que se deben mejorar, tales como los que se exponen en la tabla 4. 
Tabla 4

Factores a mejorar en el curso Teoría Contable

\begin{tabular}{lll}
\hline Factor a mejorar & Cant. & $\%$ \\
\hline Personal docente & 73 & 19 \\
\hline Estrategias y metodologías utilizadas & 65 & 17 \\
\hline Materiales y recursos académicos & 77 & 20 \\
\hline Formas de evaluación & 84 & 22 \\
\hline Mayores asesorías y horas de clase & 92 & 23 \\
\hline TOTAL & 391 & 100 \\
\hline
\end{tabular}

Fuente: elaboración propia con base en la encuesta y cálculos propios.

Lo anterior torna axiomática la necesidad de reconsiderar varios procesos en la forma, la orientación, las estrategias y la evaluación utilizadas en el mencionado espacio académico. Además, abre la ventana para pensar en reformar el currículo y privilegiar estos escenarios de formación. Sobre el particular, Muñoz, Ruiz y Sarmiento (2013) consideran que los obstáculos en la enseñanza en los desarrollos disciplinares de la contabilidad pueden encontrarse en el apego a metodologías tradicionales, en la falta de reflexión problematizadora y la ausencia de articulación entre los procesos de formación. Bajo este panorama, es pertinente entonces reflexionar en torno a lo planteado por Ordoñez (s. f., p. 10): "Es necesario repensar los cursos de teoría contable y no pretender en un semestre llenar de documentos interesantes al estudiante como si su cerebro fuera una tabula rasa".

Ahora bien, de igual forma se indagó en los encuestados sobre quienes habían reprobado el curso, encontrándose que el 17,36 \% se había inscrito por dos o más ocasiones a este (véase la figura 1). Las razones a las que atribuyeron la pérdida del curso fueron: metodología del docente, complejidad de las temáticas, estrategias de evaluación e inasistencia por desmotivación.

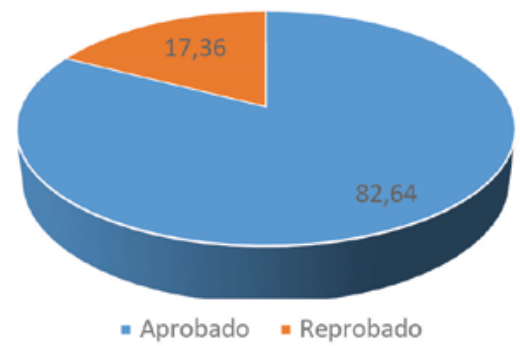

Figura 1. Relación porcentual de los estudiantes del curso de Teoría Contable que aprobaron o reprobaron.

Fuente: elaboración propia.
Igualmente, se cuestionó acerca de la posibilidad de realizar un posgrado en temas relacionados con teoría contable y la potencial vocación para desempeñar su vida profesional en el área. Los resultados se presentan en la tabla 5.

Tabla 5

Posgrado y desempeño profesional

\begin{tabular}{lcccc}
\hline \multirow{1}{*}{ Pregunta } & \multicolumn{2}{c}{ SÍ } & \multicolumn{2}{c}{ NO } \\
\cline { 2 - 6 } & Cant & \% & Cant & \% \\
\hline $\begin{array}{l}\text { ¿Le gustaría realizar un posgrado que le } \\
\text { permitiera profundizar en teoría contable? }\end{array}$ & 185 & 36,9 & 316 & 63,1 \\
\hline $\begin{array}{l}\text { ¿Le gustaría que su desarrollo } \\
\text { profesional estuviese relacionado con } \\
\text { teoría contable? }\end{array}$ & 269 & 53,7 & 232 & 46,3 \\
\hline
\end{tabular}

Fuente: elaboración propia con base en la encuesta y cálculos propios.

Respecto a la tabla 5, se halló una contradicción en los imaginarios de los estudiantes, pues pese a que el 53,7\% de los participantes afirma que le gustaría que su desarrollo profesional estuviese ligado a la teoría contable, tan solo el 36,9 \% la considera una opción de formación posgradual. Estos resultados dan cuenta de porqué a la fecha no se ha desarrollado una formación electiva o de profundización ( $\mathrm{x}$ semestre) en áreas relacionadas con la teoría contable, pues lo educandos siempre se han inclinado a tomar cursos electivos relacionados con procesos técnicos.

Así las cosas, es indudable que la teoría contable está siendo vista como un curso aislado, que versa sobre procesos complejos, largas teorías y muy distante de su verdadero rol como escenario para la edificación de una disciplina con carácter científico. Es por ello que conviene retomar lo planteado por Gómez (2006, p. 149), quien concluye:

La teoría contable como tema de abordaje o materia de estudio es compleja. En un entorno como el nuestro tampoco es fácil abordarla, enseñarla, discutirla, pues persisten concepciones pragmáticas sobre la naturaleza de la contabilidad en el colectivo contable nacional y sobre todo en la misma comunidad universitaria. Requerimos evaluar, revisar y ajustar nuestras concepciones de la contabilidad y de la Teoría Contable, así como las metodologías y enfoques con que las enseñamos o abordamos en el aula. Hemos visto el inmenso camino que implica este tema y hemos visto la necesidad de articular la teoría contable con metodologías particulares para su enseñanza. Esto genera la necesidad de una Pedagogía de la teoría contable. 


\section{Conclusiones}

La Universidad de la Amazonia, y especialmente el programa de Contaduría Pública, deben generar procesos de transformación en torno a la teoría contable, no solo como espacio académico, sino como practica transversal en el trasegar académico de los estudiantes. Esto en razón a que la teoría se considera un curso dentro de la malla curricular, lejos de su papel protagónico en la edificación de una disciplina contable basada en la investigación, el desarrollo social y el rol gerencial del contador público.

En tal sentido, se hace imperioso revisar las estrategias metodológicas, los medios y recursos educativos y las formas de evaluación, además de los contenidos curriculares, pues debe existir especial sinergia entre estos procesos a fin de fomentar verdaderos espacios de construcción y desarrollo académico, disciplinar e investigativo, pues si bien el estudiante debe generar nuevas dinámicas, es imposible desconocer el rol de los docentes y la institución en este cometido.

En términos curriculares y de estrategias didácticas, se considera importante el desarrollo de proyectos de aula integrales, es decir, que permitan el desarrollo conjunto de actividades y procesos al interior de varios espacios académicos, desligando de esta manera el modelo tradicional, y dando paso a un trabajo construido, integrado y articulado que facilite el desarrollo de competencias multidisciplinares en los futuros contadores públicos.

A manera de recomendación, la universidad, y especialmente el programa académico de Contaduría Pública, pueden generar un seminario permanente de formación en investigación y desarrollo disciplinar para los estudiantes y maestros, espacio diseñado para la convergencia académica y la generación de iniciativas que fomenten el desarrollo de competencias investigativas, argumentativas y críticas en la comunidad contable, de manera que sea este el primer paso para que la teoría contable traslape las barreras de una asignatura a todo un movimiento de pensamiento crítico y desarrollo académico.

\section{Referencias}

Ariza, Y. y Villasmil, M. (2014). Teoría contable: fundamento de análisis en el ejercicio profesional y la práctica pedagógica. Revista Desarrollo Gerencial, 6(2), 41-71.
Avellaneda, S. B. y Avellaneda, C. A. (s. f.). Globalización y educación contable, crisis y retos. En IV Encuentro nacional de profesores de Contaduría Pública. Recuperado de http:// www.conveniocpc.org/images/Memorias_iv_encuentro/ Mesa_Teoria_Contable/Globalizaci\%C3\%B3n_y_educaci\%C3\%B3n_contable_crisis_y_retos.pdf, consultado el 29.04.2016.

Barone, S.y Mella, P. (2003). Acción educativa y desarrollo humano en la universidad de hoy. Revista Iberoamericana de Educación, 31. Recuperado de http://rieoei.org/ rie31a07.htm, consultado el 30.06.2016

Beltrán, N. C., Gómez, G. y Figueroa, J. (2013). El emprendimiento empresarial en los estudiantes de Administración de Empresas de la Universidad de la Amazonia, en la creación del proyecto Empresa. Revista FACCEA, 2(3), 94-101.

Cortés, J. H. (2006). El pensamiento crítico: algunas reflexiones en torno a la educación contable. Iv Foro de Educación Contable, Universidad de Manizales, Caldas, Colombia.

Dextre, J. C. (2011). La dimensión humanística en la formación del contador público. Revista Contabilidad y Negocios, 6(11), 49-55.

Franco, R. (1997). Reflexiones contables teoría, educación y moral. Colombia: Investigar editores.

Franco, R., Ariza, D., Rojas, W., Quijano, O., Martínez, G. y Gracia, E. (2002). Del hacer al saber. Realidades y Perspectivas de la educación contable en Colombia. Popayán: Editorial Universidad del Cauca.

Giraldo, G. A. (2010). Contexto económico, ética y actitud crítica en la formación del contador público. Revista Contaduría Universidad de Antioquia, 57, 221-239.

Gómez, M. (2006). Comentarios sobre el aprendizaje-construcción de la teoría contable. Revista Lúmina, 7.

González, R. L. (2008) ¿Cuál es la importancia de la capacidad de pensamiento crítico en la enseñanza contable? Adversia, Revista virtual de estudiantes de Contaduría Pública, 3, 129-153.

Grajales, J. y Cuevas, J. J. (2010). La importancia de la formación del estudiante de Contaduría Pública en el área de Teoría Contable. El caso de la Universidad del Valle. Revista Contaduría Universidad de Antioquia, 57, 83-105

Gutiérrez, M. L. (2014). Los enfoques filosóficos de generación del conocimiento y las apuestas metodológicas que exigen [Documento PDF]. Recuperado de http://www. javeriana.edu.co/blogs/mlgutierrez/files/Enfoques-yestrategias-de-investigacion4.pdf 
López, L. M. (2012). Estándares internacionales y educación contable. Revista Apuntes del CENES, 32(55), 239-261.

Martínez, G. L. (2008). Los desafíos en la educación contable: «encrucijada de una formación monodisciplinaria en un entorno complejo e incierto». Porik An, 11, 13-72.

Marulanda, E., Piedrahita, J. V. y Quintero, O. (2011). Actitud del estudiante de Contaduría Pública frente a su formación profesional y vida laboral. Adversia, Revista virtual de estudiantes de Contaduría Pública, $8,10-25$.

Muñoz, S. M., Ruiz, G. A. y Sarmiento, H. J. (enero-diciembre, 2013). Formación para la investigación contable. Una interpretación de las intencionalidades y las metódicas desde la mirada de los profesores. Revista Lúmina, 14, 200-226.

Ordoñez, S. (s.f.). ¿Contribución de la investigación contable a la formación del contador público en Colombia? Cali: Universidad del Valle.

Ortiz, J. J. (2010). La complejidad y la teoría contable. Revista Criterio Libre, 8(13), 231-250.

Ospina, C. M. (2009). Educación Contable en Colombia. Sentires de algunos actores y la educación contable como acción educativa. Revista Contaduría Universidad de Antioquia, 55, 11-40.
Ospina, W. (1994). Los románticos y el futuro. Ciencias Económicas, 9(1), 61-76.

Pinto P, G. (2010). Hacia una metodología de la investigación contable. Revista Ciencias Económicas.

Quirós, E. H. (2006). Competencias formativas y mercado profesional contable. Revista Internacional Legis de Contabilidad \& Auditoría, 26, 143-195.

Ríos, A. G. (1998). Pedagogía, conocimiento y docencia universitaria. Revista Lúmina, 2, 123-128.

Rojas, W. (2008). Congoja por una educación contable fútil. Revista Contaduría Universidad de Antioquia, 52, 259-274.

Rojas, W. (1996). Consideraciones sobre los alcances de la enseñanza de la teoría contable. Revista Cuadernos de Administración, 173-181.

Rueda, G., Pinzón, J. E. y Patiño, R. A. (2013). Los currículos de los programas académicos de contaduría pública, tras la enseñanza de lo internacional y la globalización en la contabilidad: necesidades de ajuste más allá de respuestas técnicas. Revista Cuadernos de Contabilidad, 14(35), 639-667.

Sánchez, V., Gómez, C. A., Ortiz, D., Clavijo, T. A. y Váquiro, L. P. (2016). Percepción Social Importancia del Inglés e Inclusión de Videojuegos como Herramienta de Aprendizaje. Revista Amazonia Investiga, 5(8). rsidad de la Amazonia, Florencia, Caquetá (Colombia). 